used to determine the risk of acquiring HIV infection at 18 months among the exposed infants.

Results A total of 170 exposed infant were recruited and more than two-third of the infants were males (74.1\%) with median age was $4 \pm 3$ months. One hundred and fifty (88.2\%) received infant prophylaxis and $116(68.2 \%)$ had EID done at 6weeks. Of the 170 exposed infants $19(11.1 \%)$ were positive at 18 months final outcome of which $4(21.1 \%)$ died. Time from being confirmed HIV positive to starting ART was 4.0 \pm 2.1 months. COX regression showed that the risk of acquiring HIV infection in females (Adjusted $\mathrm{HR}=0.56,95 \% \mathrm{CI}$ 0.37-0.87), babies who received infant prophylaxis (Adjusted $\mathrm{HR}=0.5,95 \% \mathrm{CI} 0.1-0.9)$, infants whose maternal CD4 count $<500$ (Adjusted $\mathrm{HR}=1.1,95 \% \mathrm{CI} 0.4-2.6)$ and time of starting ART $>3$ months (Adjusted HR=3.5,95\%CI 1.8-11.5).

Conclusion The positivity rate among studied exposed infants was high. Continuous placement of HIV positive mothers and exposed infants on prophylaxis, timely initiation of ART will go a long way in reducing the burden of pediatric HIV.

Disclosure No significant relationships.

\section{P100 BIDIRECTIONAL ASSOCIATION BETWEEN HUMAN IMMUNODEFICIENCY VIRUS AND PSORIASIS: SYSTEMATIC REVIEW OF COHORT STUDIES}

${ }^{1} Y$ i-Yu Chen*, ${ }^{2} Y u-H s i e n$ Cheng, ${ }^{3}$ Tao-Hsin Tung. ${ }^{1}$ Kaohsiung Medical University, New Taipei City, Xinzhuang District, Taiwan; ${ }^{2}$ Kaohsiung Medical University, Taipei City, Taiwan; ${ }^{3}$ Cheng Hsin General Hospital, Taipei, Taiwan

\subsection{6/sextrans-2019-sti.293}

Background Human immunodeficiency viral (HIV) induce production of autoantibodies could attack the person's immune system. Patients with HIV infection might present with more severe complications of psoriasis, a chronic autoimmune inflammatory skin disease. But there were insufficient evidences prove the risk between HIV and psoriasis.

Objectives Systematic review was performed to explore the association between HIV and psoriasis, to provide risk assessment and improve patient outcomes.

Methods We used the search strategy: '(psoriasis OR psoriatic arthritis) AND (AIDS OR HIV OR acquired immune deficiency syndrome OR human immunodeficiency virus OR CD4 OR CCR5-delta32)' in searching the Cochrane Library, PubMed and EMBASE electronic databases without language restrictions in May 2018.

Results Four cohort study (187954 participants) were included in this review. Studies report the results of the incidences of psoriasis with patients with HIV infection. While the total incidence on current data of psoriasis was $0.46 \%$, HIV patients had higher risk than normal for developing newly diagnosed with psoriasis whether receiving substance treatment or not. However, psoriasis patients in general do not appear to have increased infection rates of HIV.

Conclusion The available evidence shows that HIV is a potential risk factor for psoriasis. The evidence at the present stage only supports the relationship between HIV infection and psoriasis, but more literature is needed to support such relevance.

Disclosure No significant relationships. CONTROL AND QUALITY OF CARE IN KAJIADO CENTRAL SUB-COUNTY

${ }^{1}$ Robert Nyamweya, ${ }^{2}$ Precious Mbabazi ${ }^{*}{ }^{3}$ Claire Barake, ${ }^{3}$ Fred Avoga, ${ }^{2} E d n a$ Anab. ${ }^{1}$ National AIDS and STI Control Programme, Strategic Information Unit, Kajiado, Kenya; ${ }^{2}$ National AIDS and STI Control Programme, Care and Treatment, Nairobi, Kenya; ${ }^{3}$ Kajiado County, Department of Health, Kajiado, Kenya

\subsection{6/sextrans-2019-sti.294}

Background Every Month, health care providers submit copies of the $\mathrm{MOH} 731$ reports to the SubCounty Health records and information officers. The data is then uploaded into the DHIS and becomes available for consumption by all the relevant stakeholders. Without a regular onsite audit of the data before uploading it, several errors may occur ranging from data entry transcription errors, arithmetic errors to outright inconsistencies with the source documents. Such errors render it unusable and incorrect. In order to correct this, Kajiado Central SubCounty, adopted a timely onsite data review and audit to identify and address the gaps reflected.

Methods The SubCounty Health management team receives and reviews the monthly reports by the fifth of every month. Any gaps identified are either corrected instantly with the staff submitting the report or an on-site mentorship report is developed. Upon uploading, the DHIS reports are downloaded and cross-checked with the $\mathrm{MoH} 731$ and the source documents for consistency.

Results After a year of instituting such reviews, the data consistency between DHIS, MOH 731 and registers improved from $45 \%$ to $90 \%$. The linkage and transitioning to ART for newly identified increased from $44.4 \%$ to $90 \%$. Additionally, Maternal and infant prophylaxis improved from 99\% and $88 \%$ to $97 \%$ and $100 \%$ respectively for those on PMTCT/ ART however due to over reporting on maternal prophylaxis after cleaning there was a decline from $99 \%$ to $97 \%$. N/B There is a graph which $\mathrm{i}$ was unable to attach here which $\mathrm{i}$ communicated same to Yurim Park.

Conclusion Regular review of monthly reports at the collection point helps to quickly identify reporting gaps. Consequently, accurate gaps identification led to the correct remedial action on the technical areas and hence improvement on the indicators.

Disclosure No significant relationships.

\section{P107 SOCIO-CULTURAL FACTORS INFLUENCING CONDOM USE INTENTIONS AMONG AFRICAN MIGRANT YOUTH IN SOUTH AFRICA}

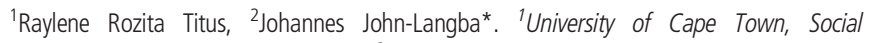
Development, Kuilsriver, South Africa; ${ }^{2}$ University of Kwazulu-Natal, Social Wotk, Durban, South Africa

10.1136/sextrans-2019-sti.295

Background Although the condom use behaviours among youth in South Africa has been studied extensivly, very little in known about the socio-cultural factors that influence intentions to use condoms during sexual intercourse among young people generally and African migrant youth in particular. This 
study utilized the theory of planned behavior to explore socio-cultural factors that influence condom use intentions among African migrant youth in South Africa.

Methods Using a qualitative research approach and snow-ball sampling procedures, indepth interviews were conducted with 20 migrant youth from other African countries, aged between 20-25 years and residing In Cape Town, South Africa.

Results Most African migrant youth residing in South Africa have acceptable levels of knowledge about condoms and condom use and linkages to HIV prevention and safer sexual practices. Traditional norms on sexual behaviour prohibit young migrants to freely discuss safer sexual choices. Gendered norms in sexual relationships, acculturation and cultural expectations of referent others reportedly influence intentions to use condom during sexual intercourse.

Conclusion The nexus among socio-cultural norms, acculturation and safer sexual choices are shown to be crucial to any HIV prevention interventions among African migrant youth in South Africa.

Disclosure No significant relationships.

\section{P109 PREVALENCE AND FACTORS ASSOCIATED WITH ANY DEPRESSIVE SYMPTOM AMONG HIV-INFECTED ADOLESCENTS IN THE REPUBLIC OF CONGO}

${ }^{1}$ Martin Herbas Ekat*, ${ }^{2}$ Marcel Yotebieng, ${ }^{3}$ Valériane Leroy. ${ }^{1}$ National AIDS Control Program, HIV Care Support Unit, Brazzaville, Congo; ${ }^{2}$ Ohio State University, Ohio, USA; ${ }^{3}$ INSERM Toulouse, Toulouse, USA

\subsection{6/sextrans-2019-sti.296}

Background To describe the frequency of any depressive symptom and associated factors among adolescents (10 - 19 years old) living with HIV in Brazzaville and Pointe Noire, Republic of Congo (RoC).

Methods Adolescents aged 10 to 19 years, on antiretroviral treatment, followed in the two Ambulatory Treatment Centers in Brazzaville and Pointe-Noire, RoC were included in this cross-sectional study. Between April 19 and July 9, 2018, faceto-face interviews were conducted with all participants using a standardized questionnaire that include the nine-item of the Patient Health Questionnaire (PHQ-9). Bivariate and multivariable log-binomial model were used to estimate the prevalence ratio (PR) and 95\% confidence interval $(95 \% \mathrm{CI})$ assessing the strength of the association between predictors and presence of depressive symptoms defined as PHQ-9 score $\geq 9$.

Results A total of 135 adolescents were interviewed. Overall, 67 (50\%) were male, 81(60\%) were 15-19 years old. PHQ-9 was $\geq 9$ among $52(39 \%)$ of participants. In bivariate analysis, the proportion of participants with PHQ-9 score $\geq 9$ was higher among participants who learnt about their HIV status after a prolonged illness; those who reported been sexually active, drinking beer, aged 15-19, stopped school, forget to take antiretroviral treatment more than 2 times in the 7 days preceding the interview, having lost both parents, and not having family support in taking ART and attending HIV care. In multivariable analysis, the following factors remained statistically significant: being 15-19 years old (PR:2.07;95\% CI:1.06-4.04), having stopped school (PR:1.60;95\%CI:1.062.42) and reporting instances of omission to take antiretroviral treatment more than 2 times in the 7 days preceding the interview (PR: 2.06;95\%CI:1.23-3.45).

Conclusion The prevalence of depressive symptoms among HIV-positive adolescents is high and is associated with older age, poor compliance, and dropping-out of school. Active screening for depression during routine medical visit and proper management is needed.

Disclosure No significant relationships.

\section{P113 RISK BEHAVIORS FOLLOWING HEPATITIS C TREATMENT AMONG GAY AND BISEXUAL MEN LIVING WITH HIV IN MELBOURNE, AUSTRALIA}

${ }^{1}$ Brendan Harney*, ${ }^{1}$ Mark Stoové, ${ }^{1}$ Rachel Sacks-Davis, 'Daniela Van Santen, ${ }^{2}$ Christopher Fairley, ${ }^{2}$ Nicholas Medland, ${ }^{3}$ Mark O'Reilly, ${ }^{4}$ Richard Moore, ${ }^{5}$ Bk Tee, ${ }^{6}$ Joseph Sasadeusz, ${ }^{7}$ David Iser, ${ }^{7}$ Janine Roney, ${ }^{8}$ Gail Matthews, ${ }^{9}$ Ed Gane, ${ }^{10}$ Maria Prins, ${ }^{1}$ Margaret Hellard, ${ }^{7}$ Joseph Doyle. 'Burnet Institute, Disease Elimination Program, Melbourne, Australia; ${ }^{2}$ Melbourne Sexual Health Centre, Melbourne, Australia; ${ }^{3}$ Prahran Market Clinic, Prahran, Australia; ${ }^{4}$ Northside Clinic, Fitzroy North, Australia; ${ }^{5}$ Centre Clinic, St Kilda, Australia; ${ }^{6}$ Royal Melbourne Hospital, Parkville, Australia; ${ }^{7}$ Alfred Health and Monash University, Department of Infectious Diseases, Melbourne, Australia; ${ }^{8}$ Kirby Institute, Sydney, Australia; ${ }^{9}$ Auckland City Hospital, Auckland, New Zealand; ${ }^{10}$ Public Health Service of Amsterdam, Amsterdam, Netherlands

\subsection{6/sextrans-2019-sti.297}

Background Hepatitis C virus (HCV) elimination among gay and bisexual men (GBM) living with HIV is feasible in many high-income countries. There is concern that risk behaviours following treatment may lead to reinfection and adversely impact HCV elimination goals. We examined risk behaviours prior to and following HCV treatment commencement among sexually active GBM living with HIV.

Methods Data were drawn from co-EC, a prospective study aiming to treat and eliminate $\mathrm{HCV}$ among people living with HIV. Pre and post-HCV treatment commencement changes in self-reported sexual and injecting drug-related behaviours among sexually active GBM attending primary and tertiary care clinics in Melbourne were assessed using McNemar's test. Modified Poisson regression with robust variance was used to examine factors associated with risk behaviours following treatment commencement.

Results Of 120 males who completed both a pre and posttreatment commencement questionnaire, 90 reported $\geq 1$ male sex partner before or after treatment commencement. Among these 90 sexually active GBM, there was no significant change pre- to post-treatment in condom-less anal intercourse with casual partners $(52.5 \% / 56.6 \%, p 0.513)$ or injecting drug use (41.2\%/45.9\%, $p$ 0.344), but a significant decrease in group $\operatorname{sex}(34.4 \% / 21.1 \%, p$ 0.011). Post-treatment commencement, condom-less intercourse (adjusted prevalence ratio (aPR) 1.80, $95 \% \mathrm{CI} 1.07-3.03, p 0.026)$ and group sex (aPR 4.53, 95\%CI 1.76-11.67, $p$ 0.002) was highest amongst those who had reported these behaviours pre-treatment. Post-treatment commencement, injecting drug use was associated with the use of crystal methamphetamine during follow-up (aPR 4.36, 95\%CI 1.27-14.94, p 0.019).

Conclusion HCV-related risk behaviours were common among sexually active GBM before and after HCV treatment and primarily occurred among the same men. There was no significant evidence of increasing risk behaviour following treatment. More frequent post-treatment $\mathrm{HCV}$ testing may be justified among GBM engaging in these behaviours to identify potential $\mathrm{HCV}$ reinfection and provide prompt re-treatment to prevent further transmission.

Disclosure No significant relationships. 